\title{
Assessment of Child Complications Related to Fluids and Electrolyte Disorders in Babel Hospital for Mothers and Children in AL-Hilla City
}

\author{
Abdul Mahdi A. Hassan \\ Assist. Prof. Ph.D., Child and Mental Health Nursing, College of Nursing, University of Babylon
}

\begin{abstract}
This is a descriptive study aimed at assessing children's complications related to fluid and electrolyte disorders. Data were collected in Babel Hospital for Maternal Children through a questionnaire model and data analysis by frequency and percentage, the results showed that $(86 \%)$ of children lose their appetite, while (14\%) They do not lose their appetite, results also show (72\%) suffer from diarrhea, while (28\%) do not suffer from diarrhea, as results show (20\%) suffer from dry mouth, while (80\%) do not suffer from dry mouth, The results also show (10\%) suffer from syncope, (90\%) do not suffer from syncope.
\end{abstract}

Objectives: Evaluation of complications related to fluid disturbances with electricity in the child.

Knowing the demographic characteristics of young children who suffer from fluids in disorders such as age, number of children in the family, and number of children in the family.

Methodology: A descriptive study used to assess complications related to a fluid electrolyte disorder for a young child. This study was conducted at Baby and Mother Hospital of Babel during the period (5/2 / 2019_3/6/2019).

A purposeful sample of (50) children, and the study was proven by a committee in the College of Nursing. Statistical SPSS was used to analyze the results of this study

Results: According to the interpretation and discussion of the study results, (72\%) of the children had diarrhea (48\%) of the children had vomiting. (62\%) are dependent on artificial feeding, (34\%) of children have experienced accidents or fall from a height.

Key words: small child, liquids, electrolyte disturbance

\section{Introduction}

Total body water (TBW) consistently represents $70 \%$ of lightweight body weight across age and sex, but differs as a percentage of actual body weight between groups and individuals due to the variance of fatty tissue deposition, which contains less water than the muscles. TBW is $-75 \%$ of total body weight in newborns, $70 \%$ in infants and $45 \%$ in the elderly. The average age of an adult male $(70 \mathrm{~kg})$ is $60 \%$ of the total body weight (42 1) as water and an adult female $(50 \mathrm{~kg})$ has $55 \%$ (27 1). This water is distributed between extracellular compartments (ECF) and intracellular (ICF). ${ }^{(1)}$
Electrolytes are chemicals in the body that regulate important physiological functions. Examples of electrolytes are sodium, chloride, magnesium, potassium, and calcium. ${ }^{(2)}$

The concentration of electrolytes in the body is controlled by a variety of hormones, most of which are made in the kidneys and adrenal glands. Sensors in specialized kidney cells monitor the amount of sodium, potassium and water in the bloodstream. ${ }^{(3)}$ Electrolyte disturbance is a defect in some ionized salts (such as bicarbonate, calcium, chloride, magnesium, phosphate, potassium and sodium) in the blood. ${ }^{(4)}$ 
Electrolyte imbalance can also be caused by a lack or excess of minerals in the body, there are medical reasons such as: Addison's disease (deceased production of hormones by the adrenal glands), alcoholism, diabetes, diarrhea, heat exhaustion, kidney disease and vomiting.

May be caused by an electrolyte imbalance with medications including:

ACE inhibitors, calcium supplements

Some of the hormones that protect potassium (which cause the kidneys to retain potassium), diuretics, which promote fluid secretion through the kidneys and potassium supplements. (5)

\section{Methodology}

1- Study design: A descriptive design used to evaluate complications related to a child's liquid electrolyte disturbances.

2- Preparing the study: This descriptive study was conducted in Baby and Mother Hospital in Babylon during the period (5/2/2019-3 / 6/2019)

3- Selecting the sample: A purposeful sample of (50) sick children.

4- Administrative permission: The study was proven by a committee in the College of Nursing.

Statistical analysis: Use descriptive statistics to analyze the results of this study.

\section{Results}

Table (1): Complication of fluid \& electrolytes disturbance

\begin{tabular}{|c|c|c|c|c|c|}
\hline \multirow{2}{*}{ No } & \multirow{2}{*}{ Variables } & \multicolumn{2}{|c|}{ Frequency } & \multicolumn{2}{|c|}{ Percentage } \\
\hline & & Yes & No & Yes & No \\
\hline 1 & Is the child suffering from diarrhea? & 36 & 14 & $72 \%$ & $28 \%$ \\
\hline 2 & Is the child suffering from vomiting? & 24 & 26 & $48 \%$ & $52 \%$ \\
\hline 3 & Is the child suffering from a fever? & 13 & 37 & $26 \%$ & $74 \%$ \\
\hline 4 & Is the child drinking adequate amounts of water? & 27 & 23 & $54 \%$ & $46 \%$ \\
\hline 5 & Is the child lost his appetite? & 43 & 7 & $86 \%$ & $14 \%$ \\
\hline 6 & Is breastfeeding a child? & 19 & 31 & $38 \%$ & $62 \%$ \\
\hline 7 & Is the sunken eyes of a child? & 32 & 18 & $64 \%$ & $36 \%$ \\
\hline 8 & Is the child suffering from lack of elasticity of the skin? & 15 & 35 & $30 \%$ & $70 \%$ \\
\hline 9 & Is the child suffering from dry mouth? & 10 & 40 & $20 \%$ & $80 \%$ \\
\hline 10 & Is the child suffering from lack of urine output? & 26 & 24 & $52 \%$ & $48 \%$ \\
\hline 11 & Is your child suffering from a lack of sweating? & 20 & 30 & $40 \%$ & $60 \%$ \\
\hline 12 & Is the child suffering from fainting ? & 5 & 45 & $10 \%$ & $90 \%$ \\
\hline 13 & Is the activity of the child changing, become idle? & 40 & 10 & $80 \%$ & $20 \%$ \\
\hline 14 & Is the color of the skin change, becoming pale? & 35 & 15 & $70 \%$ & $30 \%$ \\
\hline 15 & Is the color of urine changing? & 30 & 20 & $60 \%$ & $40 \%$ \\
\hline
\end{tabular}




\section{Discussion}

Also (48\%) of the children suffer from vomiting, while (52\%) do not suffer from this point, and excessive vomiting, especially over a long period of time, leads to the loss of excess water and electrolytes from the body, and the result agrees as my opinion.

(26\%) of children suffer from fever, while $(84 \%)$ do not suffer from this point, the temperature rises quickly or exceeds 39.5 degrees Celsius, and breathing increases, and the heart rate usually rises. Dehydration is caused by excessive sweating and increased vapor loss due to the rapid respiratory rate.

Also (38\%) of infants are breastfed, while (62\%) depend on breastfeeding, which may cause diarrhea at some time or vomiting.

$(30 \%)$ of children suffer from decreased elasticity of the skin due to fluid and electrolyte loss, while (70\%) do not suffer from this point.

Also (10\%) of children suffer from syncope there may be too much or too little sodium that can cause cell dysfunction and coma, while $(90 \%)$ do not suffer from this point.

(14\%) of children suffer from physical disabilities and diseases, while (86\%) do not suffer from them. There are diseases that cause fluid and electrolyte loss such as kidney or digestive disorders.

Also (34\%) of children experienced accidents or falls from high places. In these cases, the child experienced severe bleeding that leads to fluid and electrolyte disturbances, while $(66 \%)$ do not suffer from this point.

\section{Conclusion}

According to the interpretation and discussion of the study results, the following conclusion was concluded:
1- (72\%) of children suffer from diarrhea.

2- $(48 \%)$ of the children suffer from vomiting.

3- $(62 \%)$ depends on industrial nutrition.

4- (34\%) of the children were exposed to accidents or fall from a height.

\section{Recommendations:}

1- Baby food must contain minerals and vitamins.

2 - The child must drink a lot of fluids, and drink a lot of fluids when you have a fever, vomiting or diarrhea.

3- Protecting the child from any accidents.

Financial Disclosure: There is no financial disclosure.

Conflict of Interest: None to declare.

Ethical Clearance: All experimental protocols were approved under the College of Nursing and all experiments were carried out in accordance with approved guidelines.

\section{References}

1. Aitkenhead AR, Rowbotham DJ, Smith G. Textbook of Anesthesia, $4^{\text {th }}$ Edn. London: Churchill Livingstone, 2001.

2. Mosby's Medical Dictionary, 8th edition. (C) 2009, Elsevier.

3. Benjamin W, MD, FACEP, FAAEM. Medical Editor: Melissa Conrad Stöppler, MD, Chief Medical Editor.2000.

4. Gale Encyclopedia of Medicine. Copyright 2008 The Gale Group, Inc. 2008.

5. Cynthia Haines ,MD Last Review Date : Aug 1 ,2013 (C) 2014 Health grades operating company , Inc. 2013. 